\title{
Factores de riesgo relacionados con la prevalencia de sobrepeso y obesidad en adolescentes
}

Cúcuta, Colombia

Recibido:

21 de Julio

de 2015

Aceptado por pares:

18 de Diciembre de 2015

Aprobado:

23 de Diciembre de 2015

"Enfermera.

Doctora en

Educación

Docente

Universidad

Francisco de

Paula Santander.

Cúcuta, Colombia.

Correo electrónico:

gloriaesperanza

zp@ufps.edu.co

"*nfermera.

Enfermera Área

Gestantes. E.S.E

IMSALUD.

Cúcuta, Colombia.

Correo electrónico:

yasminufps@

hotmail.com

${ }^{* * *}$ Enfermera.

Enfermera de

Urgencias. Clínica

Santa Ana. Cúcuta

Colombia. Correo

electrónico:silvia

rodriguezberrio@

gmail.com

***** Descriptores en Ciencias de la Salud (DeCS), en la página http:// decs.bvs.br/E homepagee.htm de la Biblioteca virtual en salud del proyecto BIREME, de la Organización Mundial de la Salud y de la Organización Panamericana de Salud.

\author{
Gloria Esperanza Zambrano-Plata* \\ Yasmin Gabriela Otero-Esteban** \\ Silvia Lisbeth Rodríguez-Berrio ${ }^{* * *}$
}

\section{RESUMEN}

Objetivo: identificar los factores de riesgo relacionados con la prevalencia de sobrepeso y obesidad en adolescentes entre 10 y 18 años que pertenecen a los colegios públicos de la ciudad de Cúcuta, durante el primer semestre del 2014. Materiales y Métodos: estudio descriptivo de corte transversal; con una población: 61.172 adolescentes y una muestra de 382 estudiantes matriculados en los colegios públicos de Cúcuta. El instrumento fue diseñado por las investigadoras para valorar las características sociodemográficas, medidas antropométricas, factores endógenos, exógenos y percepción del exceso de peso y de su imagen. Resultados: el exceso de peso presento alta proporción en la adolescencia temprana y en el estrato socioeconómico 2. Predominio de sobrepeso en mujeres y obesidad en hombres. En los factores endógenos asociados con estos trastornos, prevaleció el antecedente familiar de sobrepeso y obesidad, además recibieron otra alimentación y no leche materna durante los primeros seis meses, y como antecedentes personales presentaron ansiedad y parto prematuro. En los factores exógenos la alimentación de los adolescentes con sobrepeso es alta en grasas y en los obesos es alta en azúcares, baja en verduras y fibra. Los estudiantes con déficit y con peso normal, se sienten flacos y sanos; los adolescentes con sobrepeso perciben su silueta mayor, y los obesos la perciben menor a la establecida para su índice de masa corporal. Conclusiones: los adolescentes escolarizados presentan una alta prevalencia de sobrepeso y obesidad, observándose una mayor preponderancia de factores de riesgo endógenos y exógenos. De igual manera, presentan una imagen corporal que no corresponde a su estado nutricional.

PALABRAS CLAVE: adolescentes, escolar, estado nutricional, factores de riesgo ${ }^{* * * *}$.

Para citar este artículo / To reference this article / Para citar este artigo

Zambrano-Plata GE, Otero-Esteban YG, Rodríguez-Berrio SL. Factores de riesgo relacionados con la prevalencia de sobrepeso y obesidad en adolescentes. Rev. cienc. cuidad. 2015; 12(2): 72-86. 


\section{Risk factors associated with the prevalence of overweight and obesity among adolescent}

Objective: to identify risk factors associated with the prevalence of overweight and obesity in adolescents aged 10 to 18 who belong to public schools in Cucuta city, in the first half of 2014. Materials and Methods: a descriptive cross-sectional study; population: 61,172 adolescents as a sample: 382 students enrolled in public schools in Cúcuta. The instrument was designed by the researchers to assess sociodemographic, anthropometric measurements, endogenous factors, exogenous and perception of overweight and image. Results: there was a high overweight proportion in early adolescence and socioeconomic level 2: prevalence of overweight in women and obesity in men. According to endogenous factors associated with these disorders, the prevailing factors were: family history of overweight and obesity in addition they didn 't have other food and breast milk for the first six months, and as personal history showed anxiety and prematurity. In the exogenous factors, the adolescent feeding is high in fat and in obese adolescents is high in sugar, low in vegetables and fiber. Students with deficit and normal weight, feel thin and healthy; overweight adolescents perceive their greater size shape, obese students perceive a lower size than the established one for BMI. Conclusions: school teenagers have a high prevalence of overweight and obesity, showing a greater preponderance of endogenous and exogenous risk factors. Similarly, the body image of the adolescents does not correspond to their nutritional state.

KEY WORDS: adolescents, school, nutritional state, risk factors. 
ISSN 1794-9831

E-ISSN 2322-7028

Vol. 12 No. 2

Jul - Dic 2015

Cúcuta, Colombia

\section{Os fatores de risco associados com a prevalência de sobrepeso e obesidade entre adolescentes}

\section{RESUMO}

Objetivo: identificar fatores de risco associados com a prevalência de sobrepeso e obesidade em adolescentes de 10 a 18 anos que pertencem a escolas públicas na cidade de Cucuta, no primeiro semestre de 2014. Materiais e Métodos: um estudo descritivo transversal ; População: 61,172 adolescentes mostra: 382 alunos matriculados em escolas públicas de Cúcuta. $\mathrm{O}$ instrumento foi projetado pelos pesquisadores para avaliar sociodemográficas, medidas antropométricas, fatores endógenos, exógeno e percepção de excesso de peso e sua imagem. Resultados: sobrepeso alta proporção presente no início da adolescência e estratos sócio-econômicos 2: prevalência de excesso de peso em mulheres e obesidade em homens. Em fatores endógenos associados a esses distúrbios, a história da família predominante do sobrepeso e obesidade, além recebeu nenhum outro leite alimentos e de mama para os primeiros seis meses e, como história pessoal mostrou ansiedade e prematuridade. Nos factores exógenos alimentação adolescentes com sobrepeso é rico em gordura e obesidade é rico em açúcar, pobre em vegetais e fibras. Estudantes défice e de peso normal, se sentir magra e saudável; adolescentes com sobrepeso percebem sua maior silhueta, obesos e perceber inferiores aos estabelecidos para o IMC. Conclusões: adolescentes escola tem uma alta prevalência de sobrepeso e obesidade, mostrando uma maior preponderância de fatores de risco endógeno e exógeno. Da mesma forma, uma imagem do corpo que não corresponde ao seu estado nutricional é apresentada.

PALAVRAS-CHAVE: adolescentes, escolares, estado nutricional, fatores de risco. 


\section{INTRODUCCIÓN}

S egún la Organización Mundial de la Salud (OMS) (1), la obesidad y el sobrepeso se definen como una serie de trastornos nutricionales en donde existe una acumulación anormal o excesiva de grasa en el organismo, que puede ser perjudicial para la salud.

Según estadísticas de la OMS, se encontró que el sobrepeso y la obesidad son el quinto factor principal de riesgo de defunción en el mundo; cada año fallecen por lo menos 2,8 millones de personas adultas. "En las décadas entre 1980 y 2008 la prevalencia de la obesidad se ha duplicado en el mundo, y en la actualidad más de una de cada 10 personas la padece; en total 502 millones de ciudadanos en el planeta -205 millones de hombres y 297 millones de mujeresson obesos" (2).

El exceso de peso es un problema que afecta no solamente a la edad adulta, existen numerosos estudios en donde se señala un aumento en el problema de la obesidad infantil (3-5). Al respecto la OMS en el año 2010 , encontró que alrededor de 43 millones de niños menores de cinco años de edad tenían sobrepeso, al igual que un $10 \%$ de la población de infantes en edad escolar entre 5 y 17 años (5).

La OMS declara que los niños con sobrepeso tienen muchas probabilidades de convertirse en adultos obesos en comparación con los niños sin sobrepeso (7), tienen más probabilidades de sufrir, a edades más tempranas, diabetes y enfermedades cardiovasculares (8) y exacerbación de enfermedades gastrointestinales (9) que, a su vez, se asocian con un aumento en la probabilidad de muerte prematura (10) y discapacidad (11).

Colombia no es ajena a la presencia de estos trastornos nutricionales en la población infantil. Según la Encuesta Nacional de la Situación Nutricional (12) realizada por el Instituto Colombiano de Bienestar Familiar (ICBF) en el año 2010, el 17,5 \% de los niños y jóvenes encuestados presentó exceso de peso, con una distribución de $13,4 \%$ con sobrepeso y el 4,1\% con obesidad, evidenciándose una mayor prevalencia en el área urbana (19.2\%), en comparación con la rural $(13.4 \%)$.

El Instituto Departamental de Salud (IDS) de Norte de Santander, en colaboración con el Sistema de Vigilancia Epidemiológica, Alimentaria y Nutricional (SISVAN), durante el año 2011 reveló que de 107
619 adolescentes nortesantandereanos menores de 18 años, el $34,07 \%$ tiene sobrepeso y el $16,72 \%$ posee obesidad. También expuso estadísticas a nivel municipal, en las cuales se reflejó que la Ciudad de San José de Cúcuta presentó, en este mismo año, la cifra de 37046 adolescentes menores de 18 años distribuidos en $14,82 \%$ con sobrepeso y el 7,41\% con obesidad. Se considera que los niños con estas condiciones tienen una probabilidad del $79 \%$ de riesgo de ser obesos en su edad adulta (13).

Estos trastornos nutricionales evidencian la necesidad de estudiar en la población un gran número de factores que inciden en su aparición, enmarcados en aspectos endógenos propios del organismo -como lo son la herencia y la genética- y exógenos producto del medio ambiente, los estilos de vida, el contexto sociocultural y demográfico de cada persona.

Dentro de los factores endógenos que se han relacionado con la obesidad se encuentra el género, observándose una mayor prevalencia en las mujeres (14). Igualmente, diversas investigaciones han encontrado asociación entre la obesidad y el estrés (15), la ansiedad (16), el bajo peso al nacer (17), el hipotiroidismo congénito (18), la ausencia de lactancia materna exclusiva en los primeros 6 meses de vida (19) y antecedentes familiares de sobrepeso u obesidad $(20,21)$.

Con respecto a los factores exógenos, los hábitos nutricionales tienen actualmente gran incidencia en el exceso de peso en la población escolar debido al aumento en la ingesta de comidas rápidas $(8,22)$, las cuales se caracterizan por ser hipercalóricas, ricas en grasa, sal y azúcares, pero pobres en vitaminas, minerales y otros micronutrientes saludables.

Asimismo, en los adolescentes se evidencia un mayor grado de vida sedentaria a causa de los nuevos estilos de vida, encontrándose principalmente que la tecnología es la que motiva a la población infantil a dejar de lado actividades físicas que se realizaban en tiempos pasados (23). Otros factores exógenos relacionados con el sobrepeso y la obesidad son la baja escolaridad de los padres (23) y el estrato socioeconómico bajo (24).

Otro aspecto que se ha estudiado es la percepción que tienen los adolescentes sobre su salud y su estado nutricional, observándose que, frecuentemente, los jóvenes no perciben su estado nutricional real 
Factores de riesgo relacionados con la prevalencia de sobrepeso y obesidad en adolescentes - Gloria Esperanza Zambrano-Plata, Yasmin Gabriela Otero-Esteban, Silvia Lisbeth Rodríguez-Berrio

ISSN 1794-9831

E-ISSN 2322-7028

Vol. 12 No. 2

Jul - Dic 2015

Cúcuta, Colombia ni consideran el exceso de peso como un problema de salud (25), fenómeno que se constituye en un obstáculo para la estrategias de prevención y control del sobrepeso y la obesidad.

Partiendo de la alta prevalencia de la obesidad y el sobrepeso en los adolescentes del Municipio de Cúcuta, la presente investigación busca identificar los factores de riesgo relacionados con el exceso de peso en este grupo poblacional.

\section{MATERIALES Y MÉTODOS}

Estudio con enfoque cuantitativo de tipo descriptivo, de corte transveral, en adolescentes escolarizados. La población estuvo formada por 61172 adolescentes de 10 a 18 años de edad, matriculados en los colegios públicos del municipio de Cúcuta. Se estimó una muestra de 382 estudiantes, con un error de muestreo del $5 \%$, un nivel de confianza del $98 \%$ y un nivel de heterogeneidad del $50 \%$. Se realizó un muestreo polietápico, seleccionándose al azar un colegio por cada uno de los 5 centros administrativos de educación local (CADEL); posteriormente, se realizó un muestreo proporcional por grado y mediante un muestreo aleatorio simple se seleccionaron los estudiantes de cada grado. Los criterios de inclusión definidos fueron: ser estudiantes matriculados en colegios públicos del municipio de Cúcuta y tener una edad entre 10 y 18 años.

Para la recolección de la información, las investigadoras diseñaron el instrumento Factores de Riesgo Relacionados con la Prevalencia de Sobrepeso y Obesidad, el cual constó de 16 ítems, distribuidos en cinco dimensiones: características sociodemográficas, estado nutricional, factores endógenos, factores exógenos y percepción del estado nutricional. El instrumento fue sometido a validación facial mediante evaluación por seis expertos y prueba piloto. La fiabilidad del instrumento es buena, con un alfa de cronbach de 0.7 .

El instrumento fue diligenciado de manera autoadministrada por los participantes, previo consentimiento informado por parte de los estudiantes y de su acudiente, de acuerdo con lo establecido en la Resolución 08430 de 1993 (26).

\section{OBJETIVOS}

\section{Objetivo general}

Identificar los factores de riesgo relacionados con la prevalencia de sobrepeso y obesidad en adolescentes matriculados en colegios públicos de la ciudad de Cúcuta durante el primer semestre del año 2014.

\section{Objetivos específicos}

- Caracterizar la población de estudio según variables sociodemográficas de interés.

- Estimar la prevalencia de sobrepeso y obesidad en la población objeto.

- Identificar los factores de riesgo endógenos de sobrepeso y obesidad en la población objeto.

- Identificar los factores de riesgo exógenos de sobrepeso y obesidad en la población objeto.

- Determinar la percepción que tienen los adolescentes frente a su imagen corporal.

\section{RESULTADOS}

\section{Caracterización demográfica}

La muestra de la investigación estuvo representada por 382 estudiantes matriculados en los colegios públicos del municipio de Cúcuta. Hubo un leve predominio del género masculino (51\%); el $53 \%$ de los estudiantes se encontraba en la adolescencia temprana (10 a 13 años), el $45 \%$ estaba en la adolescencia media (14 a 16 años) y un bajo porcentaje estaba en la adolescencia tardía (17 y 18 años); la edad promedio fue de 13 años. Respecto al estrato socioeconómico se identificó que la mayoría de los estudiantes eran pobres, correspondiendo al estrato $2(65 \%)$ y $1(28 \%)$.

\section{Estado nutricional}

Teniendo en cuenta que la talla para la edad (T/E) refleja el crecimiento lineal alcanzado en relación con la edad cronológica, en la muestra estudiada la mayor proporción $(79 \%)$ presentó una talla adecuada para la edad; sin embargo, se evidenció una baja proporción $(2 \%)$ de niños con baja talla para la edad, indicador que reflejó un problema nutricional crónico. Con relación al estado nutricional, se encontró que más de la mitad (55\%) de los escolares, presentaba algún tipo de trastorno nutricional, siendo más frecuente la obesidad (15\%) y el sobrepeso (29\%), tal como se aprecia en la gráfica 1 . 
Gráfica 1. Distribución del estado nutricional en estudiantes de colegios.

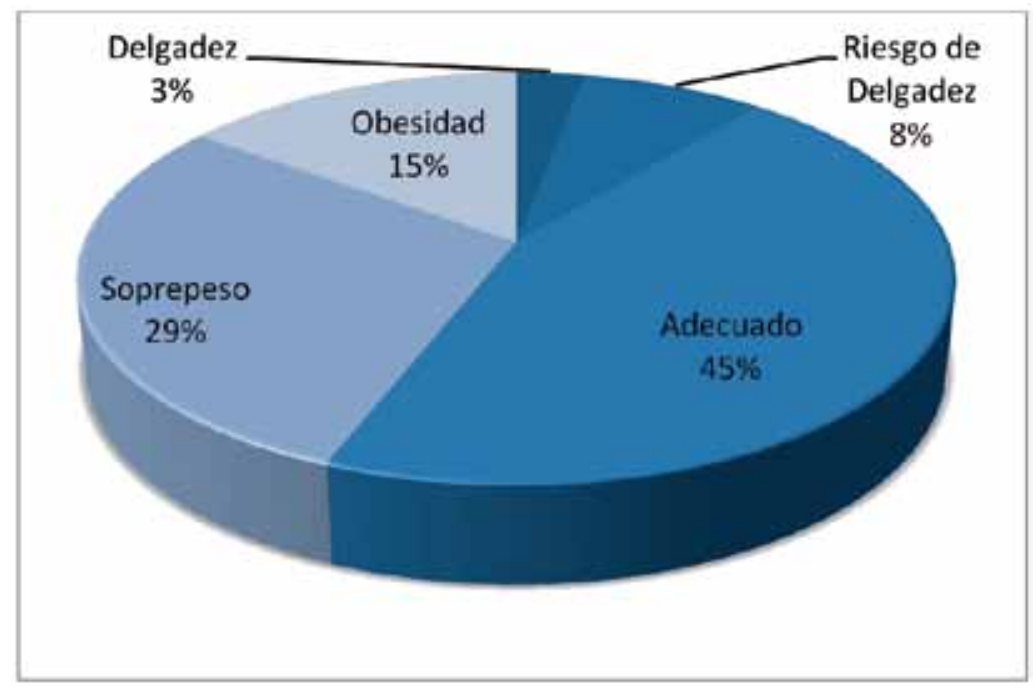

Fuente: Cuestionario Factores de Riesgo Relacionados con la Prevalencia de Sobrepeso y Obesidad en Adolescentes, 2014.
ISSN 1794-9831

E-ISSN 2322-7028

Vol. 12 No. 2

Jul - Dic 2015

Cúcuta, Colombia

\section{Factores endógenos}

Enlosestudiantesquese encontraban enlaadolescencia temprana predominó la presencia de algún trastorno nutricional, especialmente el sobrepeso $(32 \%)$ y la obesidad $(17 \%)$. En la adolescencia media y tardía existió una mayor prevalencia de peso adecuado para la edad, seguido de sobrepeso y obesidad. No se encontró asociación estadísticamente significativa entre la edad y el sobrepeso $u$ obesidad $(p=0,509)$, como se puede observar en la tabla 1.
La prevalencia de trastornos nutricionales fue mayor en los hombres (59\%) en comparación con las mujeres (51\%). Casi la mitad de las mujeres presentaban un peso adecuado y, en menor proporción, sobrepeso (31 \%) y obesidad (12\%). En los hombres se observó una mayor prevalencia de obesidad (18\%) en comparación con las mujeres. No se encontró asociación estadísticamente significativa entre el género y la presencia de sobrepeso $u$ obesidad $(\mathrm{p}=$ $0,561)$.

Tabla 1. Relación del estado nutricional y la edad en estudiantes de colegios.

\begin{tabular}{|c|c|c|c|c|c|c|}
\hline \multirow{3}{*}{$\begin{array}{l}\text { GRUPOS } \\
\text { DE EDAD } \\
\text { (AÑNOS) }\end{array}$} & \multicolumn{5}{|c|}{ ESTADO NUTRICIONAL SEGÚN IMC } & \multirow{3}{*}{$\begin{array}{c}\text { VALOR } \\
\quad P\end{array}$} \\
\hline & DELGADEZ & $\begin{array}{l}\text { RIESGO DE } \\
\text { DELGADEZ }\end{array}$ & $\begin{array}{l}\text { ADECUADO } \\
\text { PARA LA } \\
\text { EDAD }\end{array}$ & SOBREPESO & OBESIDAD & \\
\hline & $\%$ Fila & $\%$ Fila & $\%$ Fila & $\%$ Fila & $\%$ Fila & \\
\hline $10-13$ & 5 & 10 & 36 & 32 & 17 & \multirow{3}{*}{0,509} \\
\hline $14-16$ & 2 & 6 & 52 & 25 & 15 & \\
\hline $17-18$ & 0 & 4 & 64 & 28 & 4 & \\
\hline
\end{tabular}

Fuente: Cuestionario Factores de Riesgo Relacionados con la Prevalencia de Sobrepeso y Obesidad en Adolescentes, 2014. 
ISSN 1794-9831

E-ISSN 2322-7028

Vol. 12 No. 2

Jul - Dic 2015

Cúcuta, Colombia
En cuanto al orden de mayor prevalencia en las enfermedades que se relacionan a largo o corto plazo con el exceso de peso se encontró, en primer lugar, la ansiedad, con una proporción de $66 \%$, seguida por la presencia de varias de estas enfermedades con un $64 \%$; el tercer lugar fue para la prematurez, con un $57 \%$.

Mientras que la presencia de otras enfermedades se manifestó en adolescentes con sobrepeso y obesidad en un $50 \%$, el estrés se presentó con un porcentaje de $43 \%$ en adolescentes con esta misma condición nutricional y, finalmente, las condiciones como bajo peso al nacer e hipotiroidismo tuvieron un $42 \%$ y $40 \%$ respectivamente. No se encontró asociación estadísticamente significativa entre estas enfermedades y el sufrir sobrepeso $u$ obesidad $(\mathrm{p}=$ $0.122)$.

En la muestra estudiada se encontró que el $79 \%$ presentaba antecedente familiar de exceso de peso. Se pudo demostrar que existía una asociación estadísticamente significativa entre el antecedente familiar de exceso de peso y la presencia de sobrepeso y obesidad $(\mathrm{p}=0,02)$, tal como se observa en la tabla 2 .

Tabla 2. Relación del estado nutricional y el antecedente familiar de sobrepeso y obesidad en estudiantes de colegios.

\begin{tabular}{|c|c|c|c|c|c|c|}
\hline \multirow{3}{*}{$\begin{array}{c}\text { PRESENCIA } \\
\text { DE } \\
\text { SOBREPESO } \\
\text { U OBESIDAD } \\
\text { EN LA } \\
\text { FAMILIA }\end{array}$} & \multicolumn{5}{|c|}{ ESTADO NUTRICIONAL SEGÚN IMC } & \multirow{3}{*}{$\begin{array}{l}\text { VALOR } \\
\text { P }\end{array}$} \\
\hline & DELGADEZ & $\begin{array}{l}\text { RIESGO DE } \\
\text { DELGADEZ }\end{array}$ & $\begin{array}{l}\text { ADECUADO } \\
\text { PARA LA } \\
\text { EDAD }\end{array}$ & SOBREPESO & OBESIDAD & \\
\hline & $\%$ Fila & $\%$ Fila & $\%$ Fila & $\%$ Fila & $\%$ Fila & \\
\hline SI & 3 & 8 & 41 & 32 & 16 & \multirow{2}{*}{0,02} \\
\hline $\mathrm{NO}$ & 5 & 7 & 59 & 19 & 10 & \\
\hline
\end{tabular}

Fuente: Cuestionario Factores de Riesgo Relacionados con la Prevalencia de Sobrepeso y Obesidad en Adolescentes, 2014.

En relación con la cantidad de familiares con sobrepeso u obesidad se evidenció que el $53 \%$ de los adolescentes tenía dos familiares con exceso de peso, el $21 \%$, tenía solo familiar y el $15 \%$ tenía tres familiares con esta condición y muy pocos tenían cuatro o cinco familiares con sobrepeso $u$ obesidad. Dentro de estos familiares se encuentran los padres y tíos, que son de primer grado según el parentesco y en línea recta ascendente; los hermanos y abuelos, de segundo grado y colateral preferente según la línea de parentesco, y también los primos, que son de cuarto grado de consanguinidad y colateral ordinaria por línea de parentesco.

Se encontró que los estudiantes con el antecedente de lactancia materna exclusiva presentaban la mayor proporción de peso adecuado (47\%) y la menor de sobrepeso (25\%). La mayor prevalencia de obesidad $(17 \%)$ se observó en los jóvenes que reportaban el antecedente de alimentación diferente a la lactancia materna. Sin embargo, no existió asociación estadísticamente significativa entre la alimentación recibida hasta los 6 meses de edad y el hecho de sufrir sobrepeso u obesidad $(\mathrm{p}=0.235)$.

\section{Factores exógenos}

En mayor proporción, los adolescentes con sobrepeso $(59 \%)$ y obesidad $(60 \%)$ reportaban tener una dieta baja en carbohidratos. Dentro de los estudiantes que tenían un consumo alto en grasas, la mitad (50\%) correspondió a jóvenes con sobrepeso. Se observó un porcentaje importante de adolescentes con sobrepeso $(28 \%)$ y obesidad (19\%) que presentaban un alto consumo de azucares. Igualmente, se evidenció un alto consumo de lácteos y sus derivados en los escolares con sobrepeso (48 \%) y obesidad (40 \%).

Al realizar la relación entre el sobrepeso y la obesidad con el alto consumo de proteínas, los datos mostraron que el $79 \%$ de los obesos presentó una dieta hiperproteica. Con relación a los alimentos 
reguladores ricos en fibra, un alto porcentaje (35\%) de los adolescentes obesos presentaba un consumo menor a lo recomendado.

Se encontró asociación estadísticamente significativa entre la prevalencia de sobrepeso y obesidad con el consumo de cereales $(p=0.039)$, azucares $(p=0.047)$ y leche y sus derivados $(\mathrm{p}=0.000)$, mientras que para el consumo de hortalizas, verduras, leguminosas verdes, frutas, carnes, vísceras, productos elaborados, huevos, leguminosas secas, mezcla de vegetales y grasas no existe asociación estadísticamente significativa entre estos grupos de alimentos y el sobrepeso u obesidad.

Según los datos obtenidos en la variable actividad física en los adolescentes, el $34 \%$ de los estudiantes eran sedentarios, el $31 \%$ irregularmente activos y sólo el $13 \%$ de ellos eran regularmente activos; además, solamente el $22 \%$ realizaba actividad física entre 30 a 60 minutos, todos días de la semana.

La mayor proporción (49 \%) de los adolescentes activos presentó un peso adecuado para la edad, seguido de sobrepeso (34\%). En relación con los adolescentes sedentarios, predominó el peso adecuado (45 \%) seguido de la obesidad (32\%). La mayor proporción de sobrepeso (29\%) y obesidad (22\%) se presentó en los adolescentes que refirieron ser regularmente activos. No se encontró relación estadísticamente significativa entre el sobrepeso y la obesidad con la actividad física, como se aprecia en la tabla 3 .

En promedio, cada estudiante dedicaba $1.83 \pm$ 1.33 horas diarias a la realización de actividades relacionadas con actividad física, dentro de las cuales se incluía hacer deporte, salir a caminar o trotar e ir al gimnasio. Los resultados mostraron que en los adolescentes que realizaban una actividad física de 5-6 horas predominó el peso adecuado (61.9 $\%)$, seguido del sobrepeso (19\%). La proporción sobrepeso y obesidad fue mayor en los jóvenes que realizaban entre 3-4 horas de actividad física (48 $\%)$ y de $0-2$ horas (44\%). No se halló asociación estadísticamente significativa entre el tiempo diario de horas dedicadas a acciones que generan actividad física y el sobrepeso u obesidad $(\mathrm{p}=0.697)$.

Tabla 3. Relación del estado nutricional y la actividad física en estudiantes de colegios.

\begin{tabular}{|c|c|c|c|c|c|c|}
\hline \multirow{3}{*}{$\begin{array}{l}\text { ACTIVIDAD } \\
\text { FÍSICA }\end{array}$} & \multicolumn{5}{|c|}{ ESTADO NUTRICIONAL SEGÚN IMC } & \multirow{3}{*}{$\begin{array}{c}\text { VALOR } \\
\mathbf{P}\end{array}$} \\
\hline & DELGADEZ & $\begin{array}{l}\text { RIESGO DE } \\
\text { DELGADEZ }\end{array}$ & $\begin{array}{c}\text { ADECUADO } \\
\text { PARA LA } \\
\text { EDAD }\end{array}$ & SOBREPESO & OBESIDAD & \\
\hline & $\%$ Fila & $\%$ Fila & $\%$ Fila & $\%$ Fila & $\%$ Fila & \\
\hline $\operatorname{ACTIVO}(\mathrm{A})$ & 2 & 6 & 49 & 34 & 9 & \multirow{4}{*}{0,447} \\
\hline $\begin{array}{c}\text { REGULARMENTE } \\
\text { ACTIVO (A) }\end{array}$ & 4 & 6 & 39 & 29 & 22 & \\
\hline $\begin{array}{c}\text { IRREGULARMENTE } \\
\text { ACTIVO (A) }\end{array}$ & 4 & 8 & 44 & 23 & 21 & \\
\hline SEDENTARIO (A) & 3 & 10 & 45 & 32 & 10 & \\
\hline
\end{tabular}

Fuente: Cuestionario Factores de Riesgo Relacionados con la Prevalencia de Sobrepeso y Obesidad en Adolescentes, 2014.

Respecto a las acciones que no generan actividad física se encontró que cada estudiante dedicaba, en promedio, $13.8 \pm 14.9$ horas diarias a la realización de actividades sedentarias; este tiempo diario osciló entre 9 y 18 horas.

El sobrepeso y la obesidad fueron mayores en los jóvenes que realizaban actividades que no producían actividad física en un periodo de 13 y 16 horas (59\% y $62 \%$ respectivamente), seguidos de los que presentaban entre 17 y 18 horas (22 $\%$ у $33 \%$ respectivamente). Se logró identificar que la prevalencia de sobrepeso y obesidad está relacionada con el tiempo diario dedicado a este
ISSN 1794-9831

E-ISSN 2322-7028

Vol. 12 No. 2

Jul - Dic 2015

Cúcuta, Colombia 
ISSN 1794-9831

E-ISSN 2322-7028

Vol. 12 No. 2

Jul - Dic 2015

Cúcuta, Colombia tipo de actividades, es decir, a mayor tiempo diario dedicado a actividades sedentarias mayor presencia de sobrepeso u obesidad $(p=0.036)$.

El $66 \%$ de estos adolescentes con exceso de peso dedicaban entre 15 y 16 horas a estas mismas acciones, mientras que los porcentajes más bajos en los estudiantes con esta misma condición nutricional corresponden a 9 y 10 horas diarias de acciones sin actividad física.

Los datos acerca de la escolaridad del padre, relacionados con el sobrepeso, reflejaron que el mayor porcentaje (42\%) correspondió al nivel de secundaria incompleta, frente al menor porcentaje de adolescentes con sobrepeso (17\%) cuyos padres son profesionales. Entre tanto, el $29 \%$ de los estudiantes obesos tiene padre técnicos y el $10 \%$ padres con nivel de primaria incompleta.

En el caso de la escolaridad de la madre, en relación con el sobrepeso, se encontró que el $34 \%$ de los estudiantes con sobrepeso tenían madres con un nivel educativo de primaria completa, frente a un porcentaje mínimo $(19 \%)$ de estudiantes con esta misma condición con madres profesionales. En comparación con los adolescentes obesos, el $19 \%$ de ellos tenían madres con primaria incompleta y el 13 $\%$ contaba con madres profesionales.
Se observó que la proporción de casos de sobrepeso (28\%) y obesidad (14\%) es mayor en estudiantes con estrato socioeconómico bajo, comparada con aquellos que tienen estrato socioeconómico alto -en este caso, nivel o estrato 4, donde sólo se reportó un caso con sobrepeso-. Además, se evidenció que los estratos socioeconómicos bajos presentan mayor proporción en los adolescentes con exceso de peso que en los de déficit de peso; sin embargo, no se encontró asociación estadísticamente significativa entre el estrato socioeconómico y el sobrepeso u obesidad $(\mathrm{p}=0,798)$.

\section{Percepción del exceso de peso y de la imagen corporal}

A partir de los resultados obtenidos, se pudo observar que en todos los estados nutricionales, según el índice de masa corporal (IMC), se presentó en mayor prevalencia la percepción del aspecto físico como flaco. Sobre el exceso de peso, se evidenció, para los estudiantes con sobrepeso, que el $64 \%$ de ellos se consideraba flaco, el $24 \%$ percibía su aspecto físico como normal y sólo el $12 \%$ se consideraba gordo. Asimismo, en los adolescentes con obesidad, el $62 \%$ de ellos se percibía como flaco, el $28 \%$ consideraba su aspecto físico como normal y el $10 \%$ con esta misma condición nutricional se consideraba gordo, esto se aprecia en la tabla 4.

Tabla 4. Relación del estado nutricional y la percepción del aspecto físico en estudiantes de colegios.

\begin{tabular}{|c|c|c|c|c|c|c|}
\hline \multirow{3}{*}{$\begin{array}{c}\text { PERCEPCIÓN } \\
\text { DEL } \\
\text { ASPECTO } \\
\text { FÍSICO }\end{array}$} & \multicolumn{5}{|c|}{ ESTADO NUTRICIONAL SEGÚN IMC } & \multirow{3}{*}{$\begin{array}{c}\text { VALOR } \\
\mathbf{P}\end{array}$} \\
\hline & DELGADEZ & $\begin{array}{l}\text { RIESGO DE } \\
\text { DELGADEZ }\end{array}$ & $\begin{array}{l}\text { ADECUADO } \\
\text { PARA LA } \\
\text { EDAD }\end{array}$ & SOBREPESO & OBESIDAD & \\
\hline & $\%$ Columna & $\%$ Columna & $\%$ Columna & $\%$ Columna & $\%$ Columna & \\
\hline NORMAL & 25 & 36 & 30 & 24 & 28 & \multirow{3}{*}{0,000} \\
\hline FLACO & 58 & 57 & 63 & 64 & 62 & \\
\hline GORDO & 17 & 7 & 7 & 12 & 10 & \\
\hline
\end{tabular}

Fuente: Cuestionario Factores de Riesgo Relacionados con la Prevalencia de Sobrepeso y Obesidad en Adolescentes, 2014.

Para el estudio se utilizó el método propuesto por Stunkard y Stellard y modificado por Collins (27) donde se muestran figuras de siluetas corporales, tanto de varones como de mujeres, que corresponden con un IMC concreto. El análisis de los estudiantes con exceso de peso reveló que, en los adolescentes 
con sobrepeso, el $45 \%$ percibía que su imagen corporal era mayor a la establecida para su IMC, el 35 $\%$ acertó en que la imagen escogida correspondía con su IMC y solamente el $20 \%$ percibía que su imagen corporal era menor a la establecida para su IMC.

Lo contrario se encontró en los adolescentes con obesidad, de los cuales el $53 \%$ percibía que su imagen corporal era menor a la establecida para su IMC, el $28 \%$ acertó en que la imagen escogida correspondía con su IMC y sólo el 19 \% percibía que su imagen corporal era mayor a la establecida para su índice de masa corporal.

La mayoría de los estudiantes encuestados (91\%) expresa que se siente sano, en contraste con un $9 \%$ de ellos que se percibe como enfermo. Adicionalmente, el $57 \%$ consideró que ser gordo es una enfermedad. Al realizar el análisis estadístico se evidenció que existe asociación estadísticamente significativa entre la percepción de la imagen corporal según el índice de masa corporal y el sobrepeso $u$ obesidad $(p=0.00)$.

\section{DISCUSIÓN}

Los datos obtenidos sobre los adolescentes escolarizados en Cúcuta evidenciaron una alta prevalencia de sobrepeso y obesidad, situación que resulta preocupante desde el punto de vista de salud pública, teniendo en cuenta que los niños con estas condiciones tienen una probabilidad del $79 \%$ de ser obesos en su edad adulta y de acelerar la aparición de graves trastornos de salud que propiciarán la aparición de enfermedades para el resto de su vida (13).

La proporción de sobrepeso y obesidad reportada en el presente estudio es tres veces mayor a la encontrada por la Encuesta Nacional de la Situación Nutricional en Colombia (ENSIN), en un estudio realizado en el año 2010 donde se halló que el $17.5 \%$ de los niños y jóvenes entre 5 y 17 años presentó exceso de peso; el $13.4 \%$ tenía sobrepeso y el $4.1 \%$ obesidad (13). Igualmente, estos hallazgos superan los registrados a nivel departamental para el año 2011, pues en Norte de Santander se reportó una baja prevalencia de sobrepeso $(2 \%)$ y obesidad $(1 \%)$ en la población menor de 18 años (13).

Se encontró que el peso adecuado para la edad presentó mayor prevalencia en las mujeres y en los escolares que se encontraban en la etapa de la adolescencia media y tardía, mientras que en el exceso de peso se destacó la adolescencia temprana, manifestándose, en mayor proporción, el sobrepeso en mujeres y la obesidad en hombres. La preponderancia de trastornos en el estado nutricional en la adolescencia temprana también se observó en un estudio realizado en Argentina (28), en el cual se concluyó que la mayor prevalencia de sobrepeso y obesidad se presentaba en la población entre 10 y 13.9 años.

Con relación a los antecedentes personales, los más frecuentes fueron la ansiedad, seguida de la prematurez y el estrés. La prematurez está relacionada con el bajo peso al nacer, que corresponde a uno de los factores más relacionados con el sobrepeso y la obesidad (29), especialmente para sobrepeso y obesidad central en etapas posteriores de la vida (30).

Los antecedentes familiares desempeñan un papel substancial. En la muestra estudiada se presentó asociación estadísticamente significativa entre tener familiares con exceso de peso y sufrir sobrepeso u obesidad. Esta relación se observó también en Argentina, donde se evidencio que existía una relación estadísticamente significativa entre el tener algún familiar con exceso de peso y sufrir sobrepeso u obesidad (31). Resultados similares se obtuvieron en Bangladesh, donde se determinó que la presencia de algún familiar con sobrepeso aumenta tres veces el riesgo de presentar sobrepeso en los adolescentes entre 10 y 15 años (21).

Evidentemente, los antecedentes familiares desempeñan un papel importante para que los niños $\mathrm{y}$ adolescentes presenten exceso de peso. Cuando ambos progenitores son obesos, el riesgo para la descendencia es del $80 \%$; cuando sólo uno de los progenitores lo es, el riesgo desciende al $40 \% \mathrm{y}$, si ninguno de los progenitores es obeso, el riesgo en sus hijos queda entre el $3 \%$ y el $7 \%$ (32)

La lactancia materna exclusiva en los primeros seis meses de vida representa la primera experiencia nutricional del nuevo ser y sus beneficios han sido ampliamente estudiados. Al respecto, en el presente estudio, los escolares con sobrepeso y obesidad informaron recibir otro tipo de alimentación y no la leche materna, demostrando un bajo suministro de lactancia materna exclusiva e influyendo en la presencia de dichos trastornos. 
ISSN 1794-9831

E-ISSN 2322-7028

Vol. 12 No. 2

Jul - Dic 2015

Cúcuta, Colombia
Guerra et al. (32) realizaron un estudio respecto al tipo de lactancia de los escolares, encontrando que niños alimentados con fórmula eran más pesados que los que recibieron leche materna en los primeros 6 meses de vida. Esto demuestra que los niños alimentados al pecho reciben señales químicas cerebrales diferentes a las de los que reciben biberón. Estas señales permiten el desarrollo del ciclo hambre saciedad (autorregulación de la ingesta) desde los primeros momentos de la vida (33).

Asimismo, se señala que el patrón de alimentación a libre demanda favorece la adquisición de una mejor capacidad de autocontrol de la ingesta al permitir que el niño responda de forma más adecuada a las sensaciones internas de hambre y saciedad, hecho que no se produce de igual forma en la alimentación artificial (32).

El sobrepeso y su condición extrema, la obesidad, son alteraciones funcionales resultantes de una desproporción entre la cantidad de calorías que la persona adquiere con los alimentos y la cantidad que es utilizada por su organismo, lo cual genera la formación en el cuerpo de un mayor depósito de grasa. Es por esto que en el desarrollo de la obesidad inciden los hábitos de alimentación, que implican ingerir constantemente productos de alto contenido calórico, el continuo estrés y la falta de actividad (34).

Los resultados muestran que los adolescentes con exceso de peso reportan una dieta baja en carbohidratos, hiperproteica, rica en grasas y con una ingesta alta en azucares. Los adolescentes con sobrepeso mantienen una dieta diaria adecuada, tanto en hortalizas, verduras y leguminosas verdes como en fibra; en contraste, los adolescentes con obesidad presentan un consumo diario bajo en estos alimentos. La ENSIN realizada en el año 2010 revela que el consumo diario en todos los grupos de edad es menor para las verduras cocidas en comparación con las verduras crudas, comportamiento que es mayor en la polacion de 9 a 18 años (12).

Las escuelas y colegios parecen ser el lugar adecuado para promover y facilitar el desarrollo del ejercicio físico en los niños y adolescentes, debido a que la mayor parte del tiempo permanecen allí; sin embargo, esto no se observa en el presente estudio, puesto que más de la mitad de la muestra estudiada no realiza actividad física, los adolescentes con exceso de peso dedican diariamente una gran cantidad de horas -13 a 16- a acciones que no producen actividad física como ver televisión, escuchar música, dormir, navegar en internet, videojuegos o quehaceres domésticos $\mathrm{y}$, en contraste, dedican pocas horas al día (3 a 4) a realizar deportes, gimnasio, caminar o trotar, lo cual influye a una vida sedentaria y múltiples complicaciones en la salud.

Esta situación también se observó en un estudio realizado en México por Trejo et al. (35), en donde los escolares no cumplen con los criterios establecidos sobre actividad física para este grupo de edad, que corresponde a 60 minutos diarios de actividad física, de moderada a intensa. Lo anterior puede asociarse aque, para los padres, es más seguro tener a sus hijos en casa que promover actividades al aire libre; sin embargo, también denota el riesgo en que se encuentran los niños para desarrollar exceso de peso, existiendo la posibilidad de continuar con un estilo de vida sedentario en la edad adulta.

Guerra et al. (32) descubrió que los estudiantes que admitieron no efectuar ninguna actividad física tienen cinco veces más riesgo de presentar exceso de peso. La prevalencia de obesidad entre adolescentes que no practican ningún deporte habitualmente es más elevada en comparación con los que sí tienen este hábito, especialmente entre los que practican actividades deportivas tres veces por semana (36).

Se halló una menor escolaridad en los padres de los adolescentes con sobrepeso y obesidad, en quienes predominó la secundaria incompleta para el padre y primaria incompleta para la madre. Estos datos son similares a los del estudio Aladino: Alimentación, Actividad Física, Desarrollo Infantil y Obesidad (37), donde se evidencia que el nivel educativo de los progenitores influye en que los niños puedan presentar o no obesidad. Un $41 \%$ de los hijos de padres con estudios universitarios tiene sobrepeso $\mathrm{u}$ obesidad frente a un $47.9 \%$ de los que tienen estudios secundarios y un $47.6 \%$ de los que sólo han alcanzado los estudios primarios.

Los ingresos familiares miden diferentes aspectos en la calidad de vida, tales como la satisfacción de las necesidades básicas, siendo una de ellas la nutrición. A través del análisis de los datos, este estudio logró determinar que el sobrepeso es mayor en los adolescentes de estrato bajo y la obesidad predomina 
en el estrato medio. Datos semejantes fueron encontrados en el estudio realizado por Santos (36), donde se evidenció que la prevalencia de obesidad es más elevada en los jóvenes pertenecientes a un nivel socioeconómico bajo.

Sobre la autopercepción de la imagen corporal, se encontró que la mayor proporción de adolescentes se percibe como flaco, tanto quienes se encuentran con déficit como los de peso normal y en exceso. Estos hallazgos son similares a los reportados en México por Villanueva y Ramírez (25), en donde el 86 \% de los adolescentes se percibe delgado. Al analizar la imagen corporal en los adolescentes con sobrepeso, la mayor proporción se percibe con una silueta mayor a la establecida para su IMC, contrario a aquellos adolescentes con obesidad, quienes perciben que su imagen corporal es menor a la establecida para su IMC. Este fenómeno también se observó en adolescentes obesos en Portugal, donde solamente el $12.7 \%$ se consideraba obeso (38).

\section{CONCLUSIONES}

Se evidenció la alta prevalencia de sobrepeso (29\%) y obesidad (15\%) en estudiantes adolescentes de colegios públicos en Cúcuta, reflejando un problema alarmante de salud pública debido a las implicaciones que el exceso de peso trae para la salud y la calidad de vida en esta población.

Dentro de los factores endógenos se evidencio que el exceso de peso es mayor en la adolescencia temprana (49\%) y en el género masculino (46\%). Otros factores de alta prevalencia en los escolares con exceso de peso son los antecedentes familiares de exceso de peso (48 \%) y la ausencia de lactancia materna en los primeros seis meses de vida (54\%).

Los factores exógenos prevalentes en los estudiantes con exceso de peso son: la baja escolaridad de los padres de los adolescentes con sobrepeso (34\%) y con obesidad (19\%), el estrato socioeconómico bajo (42\%), la dieta rica en azucares pero baja en fibra e hiperproteica, ser regularmente activos y dedicar la mayor parte de su tiempo a acciones que no generan actividad física.

Fue baja la proporción de adolescentes con sobrepeso $(12 \%)$ y obesidad $(10 \%)$, que se perciben con exceso de peso; la mayoría de ellos se percibe más delgados de lo son, de acuerdo con su estado nutricional.

\section{CONFLICTO DE INTERESES}

Los autores declaran no tener ningún conflicto de intereses.
ISSN 1794-9831

E-ISSN 2322-7028

Vol. 12 No. 2

Jul - Dic 2015

Cúcuta, Colombia

a


ISSN 1794-9831

E-ISSN 2322-7028

Vol. 12 No. 2

Jul - Dic 2015

Cúcuta, Colombia

\section{REFERENCIAS BIBLIOGRÁFICAS}

1. Organización Mundial de la Salud (OMS). Temas de salud. Obesidad. [Internet]. Ginebra: OMS; [consultado 24 de julio de 2013]. Disponible en: http://www.who.int/topics/obesity/es/

2. Lantigua IF. El mapa global de la obesidad. El Mundo (es) [Internet]. Viernes 4 de febrero de 2011; Cifras [consultado 27 julio de 2013]. Disponible en: http://www.elmundo.es/elmundosalud/2011/02/03/nutricion/1296763533.html

3. López-Villalta Lozano MJ, Soto-González A. Actualización en Obesidad. Cad Aten Primaria [Internet]. 2010 [consultado 30 de julio de 2013];17:101-107. Disponible en: http://www.agamfec.com/pdf/CADERNOS/VOL17/ vol_2/Para_saber_de_01_vol17_n2.pdf

4. Riaño-Galán G. Simposio I. Avances en el manejo del sobrepeso y de la obesidad. Sobrepeso y obesidad en la adolescencia. Nuestra realidad. BOL PEDIATR [Internet]. 2007 [consultado 30 de julio de 2013]; 47(Supl.1):8-12. Disponible en: http://www.sccalp.org/boletin/47_supl1/BolPediatr2007_47_supl1_008-012.pdf

5. Organización Mundial de la Salud (OMS). Obesidad y sobrepeso. [Internet]. Ginebra: OMS; 2012 [consultado 27 de julio de 2013]. Disponible en: http://www.who.int/mediacentre/factsheets/fs311/es/index.html

6. Olaiz-Fernández G, Rivera-Dommarco J, Shamah-Levy T, Rojas R, Villalpando-Hernández S, Hernández-Avila M, et al. Encuesta Nacional de Salud y Nutrición. $1^{a}$ ed. [Internet]. México: Instituto Nacional de Salud Pública; 2006 [consultado 24 de julio de 2013]. Disponible en: http://ensanut.insp.mx/informes/ensanut2006.pdf

7. Organización Mundial de la Salud (OMS). Datos y Cifras. 10 datos sobre la obesidad. [Internet]. Ginebra: OMS; [consultado 30 de abril de 2014]. Disponible en: http://www.who.int/features/factfiles/obesity/facts/es/index2.html

8. Moreno-Aznar LA, Alonso-Franch M. Obesidad. Protocolos diagnósticos-terapeuticos de Gastroenterología, Hepatología y Nutrición Pediátrica SEGHNP-AEP [Internet]. 2010 [consultado 4 de agosto de 2013]; (6): 319323. Disponible en: http://www.aeped.es/sites/default/files/documentos/obesidad.pdf

9. Ceruelo E, De La Fuente A, García C, De Toledo J, Lorente A, Martínez M, et al. Nutrición del niño mayor. Obesidad, Guías de actuación conjunta Pediatría Primaria-Especializada [Internet]. Madrid: Grupo de Gastroenterología Pediátrica Zona Suroeste de Madrid; 2014 [consultado 30 de abril de 2014]; 43 (20 - 23). Disponible en: http:// www.ampap.es/documentacion/protocolos/Obesidad_2012.pdf

10. Shelley L. Surgeon General Warns of Obesity Epidemic: As many as 300,000 deaths each year related to being overweight Club Industry [Internet]. 2002 [consultado 18 de diciembre de 2015];18(2):10. Disponible en: http:// search.proquest.com/docview/218317717? accountid=43636

11. Walter S, Kunst A, Mackenbach J, Hofman A, Tiemeier H. Mortality and disability: The effect of overweight and obesity. International Journal of Obesity [Internet]. 2009 [consultado 18 de diciembre de 2015]; 33(12):1410-8. Disponible en: http://dx.doi.org/10.1038/ijo.2009.176

12. Instituto Colombiano de Bienestar Familiar (ICBF). Encuesta Nacional de la Situación Nutricional en Colombia 2010. [Internet]. Bogotá: ICBF; 2010 [consultado 28 de julio de 2013]. Disponible en: http://www.icbf.gov.co/ portal/page/portal/Descargas1/Resumenfi.pdf

13. Instituto Departamental de Salud (IDS). Sistema de Vigilancia Epidemiológica Alimentaria y Nutricional (SISVAN). Diagnostico nutricional vigencia 2011 [Internet]. Cúcuta: Gobernación de Norte de Santander; 2011 [consultado 27 julio de 2013]. Disponible en: salasituacionalidsnds.weebly.com/uploads/1/0/7/1/10714324/ diagnostico_nutricional_sisvan_2011.pdf

14. Morales-González JA. Obesidad un enfoque multidisciplinario. $1^{\mathrm{a}}$ ed. [Internet]. Madrid: Universidad Autónoma del Estado de Hidalgo; 2010 [consultado 30 de enero de 2014]; Disponible en: http://www.uaeh.edu.mx/ investigacion/productos/4823/libro_de_obesidad.pdf

15. Nishitani N, Sakakibara H. Relationship of obesity to job stress and eating behavior in male Japanese workers. Int J Obes. [Internet]. 2006 [consultado 18 de diciembre de 2015];30(3):528-33. Disponible en: http://dx.doi. org/10.1038/sj.ijo.0803153

16. Gariepy G, Nitka D, Schmitz N. The association between obesity and anxiety disorders in the population: a systematic review and meta-analysis. Int J Obes. [Internet]. 2010 [consultado 18 de diciembre de 2015]; 03; 34(3):407-19. Disponible en: http://dx.doi.org/10.1038/ijo.2009.252

17. Casey PH, Bradley RH, Whiteside-mansell L, Barrett K, Gossett JM, Simpson PM. Evolution of obesity in a low birth weight cohort. Journal of Perinatology [Internet]. 2012 [consultado 18 de diciembre de 2015];32(2):91-6. Disponible en: http://dx.doi.org/10.1038/jp.2011.75

18. Wong SC, Ng SM, Didi M. Children with congenital hypothyroidism are at risk of adult obesity due to early adiposity rebound. Clin Endocrinol (Oxf). [Internet]. 2004 [consultado 18 de diciembre de 2015];61(4):441-446. Disponible en: http://dx.doi.org/10.1111/j.1365-2265.2004.02116.x 
19. Guerra C, Vila J, Apolinaire J, Cabrera A, Carballosa I, Almaguer P. Factores de riesgo asociados a sobrepeso y obesidad en adolescentes. Revista Electrónica de las Ciencias Médicas en Cienfuegos Medisur[Internet]. 2009 [consultado 30 de julio de 2013];7(2): 25-34. Disponible en: http://scielo.sld.cu/pdf/ms/v7n2/v7n2a632.pdf

20. Wojcicki J, Schwartz N, Jiménez-Cruz A, Bacardi-Gascon M, Heyman MB. Acculturation, dietary practices and risk for childhood obesity in an ethnically heterogeneous population of latino school children in the San Francisco Bay area. Journal of Immigrant and Minority Health [Internet]. 2012 [consultado 18 de diciembre de 2015];14(4):533-9. Disponible en: http://www.ncbi.nlm.nih.gov/pmc/articles/PMC4456089/

21. Bhuiyan M, Zaman S, Ahmed T. Risk factors associated with overweight and obesity among urban school children and adolescents in Bangladesh: a case-control study. BMC Pediatrics [Internet]. 2013 [consultado 18 de diciembre de 2015];13:72. Disponible en: http://search.proquest.com/docview/1353014365?accountid=43636

22. Andegiorgish AK, Wang J, Zhang X, Liu X, Zhu H. Prevalence of overweight, obesity, and associated risk factors among school children and adolescents in Tianjin, China. Eur J Pediatr. [Internet]. 2012 [consultado 18 de diciembre de 2015];171(4):697-703. Disponible en: http://dx.doi.org/10.1007/s00431-011-1636-x

23. Yi X, Yin C, Chang M, Xiao Y. Prevalence and risk factors of obesity among school-aged children in xi' an, China. Eur J Pediatr [Internet]. 2012 [consultado 18 de diciembre de 2015]; 171:389-94. Disponible en: http://search. proquest.com/docview/1095597731/fulltextPDF/4B8B98BC01DB417DPQ/3?accountid=43636

24. Lee H, Andrew M, Gebremariam A, Lumeng J, Lee JM. Longitudinal associations between poverty and obesity from birth through adolescence. Am J Public Health [Internet]. 2014 [consultado 18 de diciembre de 2015];104(5):e706. Disponible en: http://search.proquest.com/docview/1524713366?accountid $=43636$

25. Villanueva-Sánchez J, Ramírez-Moreno E. Factores asociados al sobrepeso en estudiantes de 8 a 18 años de áreas suburbanas, Hidalgo, México. Revista de la Facultad de Salud Pública y Nutrición UANL [Internet]. 2004 [consultado 4 de agosto 2013];5(3). Disponible en: http://www.respyn.uanl.mx/v/3/articulos/factores.htm

26. República de Colombia. Ministerio de Salud. Resolución 8430 de 1993, octubre 4, por la cual se establecen las normas científicas, técnicas y administrativas para la investigación en salud. Bogotá: Ministerio de Salud; 1993.

27. Collins ME. Body figure perceptions and preferences among preadolescent children. International Journal of Eating Disorders [Internet]. 1991 [consultado 30 de enero 2014]; 10(2):199-208. Disponible en: http://onlinelibrary.wiley. com/doi/10.1002/1098-108X(199103)10:2\%3C199::AID-EAT2260100209\%3E3.0.CO;2-D/abstract

28. KovalskysI, Bay L, Herscovici CR, Berner E. Prevalencia de obesidad en una población de 10 a 19 años en la consulta pediátrica. Rev Chil Pediatr [Internet]. 2005 [consultado 30 de abril de 2014]; 76 (3): 324-325. Disponible en: http://www.scielo.cl/scielo.php?script=sci_arttext\&pid=S0370-41062005000300016

29. Manuel-Apolinar L, Hernández-Valencia M. Neurobiología del bajo peso al nacer y su asociación con la diabetes tipo 2. Perinatol Reprod Hum [Internet]. 2008 [consultado 30 de enero 2014];22(2):155-164. Disponible en: http:// www.sumedico.com/documentos/26_documento.pdf

30. Perea-Martínez A, Bárcena-Sobrino E, Rodríguez-Herrera R, Greenawalt-Rodríguez S, Carbajal-Rodríguez L, Zarco-Román L. Obesidad y comorbilidades en niños y adolescentes asistidos en el Instituto Nacional de Pediatría. Acta Pediatr Mex. [Internet]. 2009 [consultado 30 de enero de 2014]; 30(3):167-74. Disponible en: http://www. medigraphic.com/pdfs/actpedmex/apm-2009/apm093g.pdf

31. Bassan ND, Solís C, Soldano OR, Vinuesa MA. Sobrepeso y obesidad en escolares de rosario Argentina relevancia de los factores hereditarios. REV. MÉD. ROSARIO [Internet]. 2011 [consultado 30 de octubre de 2013]; 77: 130136. Disponible en: http://www.circulomedicorosario.org/Upload/Directos/Revista/ad9526Bassan.pdf

32. Guerra C, Vila J, Apolinaire J, Cabrera A, Santana I, Almaguer P. Factores de riesgo asociados a sobrepeso y obesidad en adolescentes. Medisur [Internet]. 2009 [consultado 30 de octubre de 2013]; 7(2): 25-34. Disponible en: http://scielo.sld.cu/pdf/ms/v7n2/v7n2a632.pdf

33. Subcomisión de Epidemiología y Comité de Nutrición. Consenso sobre factores de riesgo de enfermedad cardiovascular en pediatría. Obesidad. Arch.argent.pediatr [Internet]. 2005 [consultado 3 de agosto de 2013]; 103(3): 262-281. Disponible en: http://www.scielo.org.ar/pdf/aap/v103n3/v103n3a13.pdf

34. Morales-González JA. Obesidad un enfoque multidisciplinario. [Internet]. Pachuca, México: Universidad Autónoma del Estado de Hidalgo; 2010 [consultado 30 de enero de 2014]. Disponible en: http://www.uaeh.edu. $\mathrm{mx} /$ investigacion/productos/4823/libro_de_obesidad.pdf

35. Trejo-Ortiz PM, Jasso-Chairez S, Mollinedo- Montaño FE, Lugo-Balderas LG. Relación entre actividad física y obesidad en escolares. [Internet]. Zacatecas, México: Universidad Autónoma de Zacatecas; 2011 [consultado 4 de agosto de 2013]. Disponible en: http://bvs.sld.cu/revistas/mgi/v28n1_12/mgi05112.htm

36. Santos-Muñoz S. La educación física escolar ante el problema de la obesidad y el sobrepeso. Rev.int.med.cienc. act.fís.deporte [Internet]. 2005 [consultado 30 de octubre de 2013]; 5(19):179-199. Disponible en: http://cdeporte. rediris.es/revista/revista19/artobesidad10.pdf 
37. Gobierno de España. Estudio ALADINO: Estudio de Vigilancia del Crecimiento, Alimentación, Actividad Física, Desarrollo Infantil y Obesidad en España. 2011. [Internet]. Madrid: Agencia Española de Seguridad Alimentaria y Nutrición. Ministerio de Sanidad, Servicios Sociales e Igualdad; 2013: [consultado 30 de octubre de 2013]. Disponible en : http://www.observatorio.naos.aesan.msssi.gob.es/docs/docs/documentos/estudio_ALADINO.pdf

38. De Sousa-Lopes PM. Body-image and obesity in adolescence: a comparative study of social-demographic, psychological, and behavioral aspects. The Spanish Journal of Psychology [Internet]. 2008 [consultado 18 de diciembre de 2015];11(2):551-63. Disponible en: http://search.proquest.com/docview/274589631?account $\mathrm{id}=43636$ 